\title{
Radar Detection using Sparsely Distributed Apertures in Urban Environment
}

\author{
Il-Young Son ${ }^{a}$, Trond Varslot ${ }^{a}$, Can Evren Yarman ${ }^{a}$, Ali Pezeshki ${ }^{b}$, Birsen Yazici ${ }^{a}$ and \\ Margaret Cheney ${ }^{a}$ \\ ${ }^{a}$ Rensselaer Polytechnic Institute, Troy, NY \\ ${ }^{b}$ Princeton University, Princeton, NJ
}

\begin{abstract}
We present a new receiver design for spatially distributed apertures to detect targets in an urban environment. A distorted-wave Born approximation is used to model the scattering environment. We formulate the received signals at different receive antennas in terms of the received signal at the first antenna. The detection problem is then formulated as a binary hypothesis test. The receiver is chosen as the optimal linear filter that maximizes the signal-to-noise ratio (SNR) of the corresponding test statistic. The receiver operation amounts to correlating a transformed version of the measurement at the first antenna with the rest of the measurements. In the freespace case the transformation applied to the measurement from the first antenna reduces to a delay operator. We evaluate the performance of the receiver on a real data set collected in a multipath- and clutter-rich urban environment and on simulated data corresponding to a simple multipath scene. Both the experimental and simulation results show that the proposed receiver design offers significant improvement in detection performance compared to conventional matched filtering.
\end{abstract}

Keywords: Radar detection, Distributed antenna, Radar data processing, Statistical signal processing

\section{INTRODUCTION}

Conventional matched filtering is widely used for radar detection. In this approach the radar measurement is matched to a known illumination template. In many situations the illumination template is unknown, due to many factors such as re-radiation from metallic objects, multiple scattering, etc. or due to the fact that the transmitted waveform is not known, as is the case in passive radar applications.

In this paper, we present a new receiver design which does not require knowledge of the illumination template; rather a transformed version of the measurement from one of the receive antennas is used as a template for matching. The scattering is modelled by a distorted-wave Born approximation using Green's functions for the environment. This model is used to express the radar measurements at different antenna elements in terms of the radar measurement at the first antenna. A binary hypothesis test for detection is then formulated and the optimal linear receiver is derived by maximizing the SNR of the corresponding test statistic. The processing at the receiver amounts to correlating a transformed version of the measurement at the first antenna with the rest of the measurements. In the free-space case, the receiver reduces to a delay-correlator that is similar to the the one reported in Attia and Steinberg. ${ }^{1}$

Passive radar systems were previously studied by Griffiths and Baker ${ }^{2,3}$ and Kulpain. ${ }^{4}$ Passive coherent location radar systems considered by Griffiths and Baker consist of cross-correlating the measurement with a "reference" direct signal (i.e., signal received from illuminator of opportunity in absence of a target). Kulpa considered correlating received signals at a pair of receivers, with time and Doppler delays for some fixed time window based on the amount of time the target remains in a range-cell. The analysis is done using a rangeDoppler cross-correlation function similar to the classic cross-ambiguity function. Thus, the model assumes free-space Green's function. Kulpa identifies several limitations of this system including difficulties due to clutter and difficulty in selecting the time window for which target remains within resolution cell. The crosscorrelation of measurements are also central to the passive imaging techniques studied previously. ${ }^{5-7}$ Norton et. al. ${ }^{5}$ considers an imaging scheme for seismic imaging application where cross-correlation of each pair of

Signal Processing, Sensor Fusion, and Target Recognition XVI, edited by Ivan Kadar, Proc. of SPIE Vol. 6567, 65671Q, (2007) - 0277-786X/07/\$18 - doi: 10.1117/12.720069 
(possibly filtered) measurements is used. These cross-correlations are backprojected to form a reconstruction along hyperbolic paths. The work by Larose et. al. and Snieder ${ }^{6,7}$ considers how cross-correlation can extract the Green's function for the environment. Matched field processing is a well-known method in the acoustic literature. The method is similar to matched filter method in the radar literature in that it relies on a template based on the incident field. The incident field is computed from the known environment model. In paper by Tolstoy ${ }^{8}$ it was shown experimentally that matched-field methods are highly sensitive to model errors. Krolik ${ }^{9}$ describes a beamforming-based method for making matched-field technique robust was explored. The method is called MV-EPC and is based on designing a robust beamformer over a range of random perturbations of the environment parameters, taking into account first- and second-order statistics of the perturbation. The method is robust as long as the perturbations are moderate. A spatial correlation technique has also been previously applied to waveform design. ${ }^{10}$

The performance of the proposed receiver is compared against conventional matched filtering on a real data set. The data set used in this paper was collected in a clutter-rich urban enivronment. Our preliminary results indicate that employing the proposed receiver can significantly improve the detection performance, quantified by the SNR of the test statistic and the area under the ROC curve (AUC).

\section{PROBLEM STATEMENT}

In this section we derive a binary hypothesis test for target detection using $n$ identical radar receive antennas. We consider a general scattering environment modelled using a Green's function and an unknown point target. This scattering model applies to free space as well as the urban environment. An underlying assumption is that the Green's function is known or can be estimated.

The Green's function is defined as the solution of the wave equation with an impulse source:

$$
\nabla^{2} g-\frac{1}{c^{2}} \ddot{g}=\delta(t) \delta(|\mathbf{x}-\mathbf{y}|) .
$$

Thus, the Green's function $g(\mathbf{x}, \mathbf{y}, t)$ represents the response observed at location $\mathbf{x}$ and time $t$, if a unit impulse is radiated from location $\mathbf{y}$ at time 0 . In this paper, we use a scalar model of the wave.

Let $E^{\text {in }}(\mathbf{y}, t)$ be the scalar electric field incident on a target. This field induces the radar scattering measured by the $i$ th receive element at position $\mathbf{x}_{i}$, which is approximately ${ }^{11}$

$$
m_{i}(t)=\iint g\left(\mathbf{x}_{i}, \mathbf{y}, t-\tau\right) T(\mathbf{y}) \ddot{E}^{\text {in }}(\mathbf{y}, \tau) d \mathbf{y} d \tau+n_{i}(t)
$$

Here, $T(y)$ is the target response at location $y$ and $n_{i}(t)$ is an additive white Gaussian noise with variance $\sigma_{i}^{2}$. For

simplicity and without loss of generality, we take the noise power at different receivers to be identical $\left(\sigma_{i}^{2}=\sigma_{n}^{2}\right.$, $i=1, \ldots, n)$. The approximation made in (2) is that the target does not induce multiple scattering effects, called the distorted-wave Born approximation.

For a point scatterer at position $\mathbf{y}=\mathbf{y}_{o}$ the radar measurement equation (2) may be expressed in the frequency domain as

$$
\hat{m}_{i}(\omega)=\omega^{2} \hat{g}\left(\mathbf{x}_{i}, \mathbf{y}_{o}, \omega\right) T\left(\mathbf{y}_{o}\right) \hat{E}^{\mathrm{in}}\left(\mathbf{y}_{o}, \omega\right)+\hat{n}_{i}(\omega)
$$

where $\hat{m}_{i}(\omega), \hat{g}\left(\mathbf{x}_{i}, \mathbf{y}_{o}, \omega\right), \hat{E}^{\mathrm{in}}\left(\mathbf{y}_{o}, \omega\right)$, and $\hat{n}_{i}(\omega)$ are Fourier transforms of $m_{i}(t), g\left(\mathbf{x}_{i}, \mathbf{y}_{o}, t\right), E^{\text {in }}\left(\mathbf{y}_{o}, t\right)$, and $n_{i}(t)$.

The radar measurement $\hat{m}_{2}(\omega)$ at receiver 2 may be expressed in terms of $\hat{m}_{1}(\omega)$, the radar measurement at receiver 1 , as

$$
\hat{m}_{2}(\omega)=\frac{\hat{g}\left(\mathbf{x}_{2}, \mathbf{y}_{o}, \omega\right)}{\hat{g}\left(\mathbf{x}_{1}, \mathbf{y}_{o}, \omega\right)} \hat{m}_{1}(\omega)-\frac{\hat{g}\left(\mathbf{x}_{2}, \mathbf{y}_{o}, \omega\right)}{\hat{g}\left(\mathbf{x}_{1}, \mathbf{y}_{o}, \omega\right)} \hat{n}_{1}(\omega)+\hat{n}_{2}(\omega) .
$$

More generally, we may express the measurement at receivers 2 to $n$ in terms of the measurement at receiver 1 by vectorizing (4) as

$$
\mathbf{m}=\mathbf{g} \hat{m}_{1}(\omega)-\mathbf{g} \hat{n}_{1}(\omega)+\mathbf{n}(\omega)
$$


where

$$
\begin{aligned}
\mathbf{m}=\left[\begin{array}{c}
\hat{m}_{2}(\omega) \\
\hat{m}_{3}(\omega) \\
\vdots \\
\hat{m}_{n}(\omega)
\end{array}\right], \quad \mathbf{g}=\frac{1}{\hat{g}\left(\mathbf{x}_{1}, \mathbf{y}_{o}, \omega\right)}\left[\begin{array}{c}
\hat{g}\left(\mathbf{x}_{2}, \mathbf{y}_{o}, \omega\right) \\
\hat{g}\left(\mathbf{x}_{3}, \mathbf{y}_{o}, \omega\right) \\
\vdots \\
\hat{g}\left(\mathbf{x}_{n}, \mathbf{y}_{o}, \omega\right)
\end{array}\right], \\
\text { and } \\
\quad \mathbf{n}(\omega)=\left[\begin{array}{c}
\hat{n}_{2}(\omega) \\
\hat{n}_{3}(\omega) \\
\vdots \\
\hat{n}_{n}(\omega)
\end{array}\right] .
\end{aligned}
$$

This shows that in the presence of a point scatterer the radar measurements are related through the linear model in (5). Thus, the problem of detecting a point scatterer may be posed as the following binary hypothesis test

$$
\begin{aligned}
& \mathcal{H}_{1}: \quad \mathbf{m}=\mathbf{g} \hat{m}_{1}+\tilde{\mathbf{n}} \\
& \mathcal{H}_{0}: \quad \mathbf{m}=\mathbf{n},
\end{aligned}
$$

or equivalently

$$
\begin{array}{ll}
\mathcal{H}_{1}: & \mathbf{m} \mid \hat{m}_{1} \sim C N\left(\mathbf{g} \hat{m}_{1}, \mathbf{R}_{\tilde{\mathbf{n}} \tilde{\mathbf{n}}}=\sigma_{n}^{2} \mathbf{g g}^{H}+\sigma_{n}^{2} \mathbf{I}\right) \\
\mathcal{H}_{0}: & \mathbf{m} \sim C N\left(\mathbf{0}, \mathbf{R}_{n n}=\sigma_{n}^{2} \mathbf{I}\right),
\end{array}
$$

where

$$
\tilde{\mathbf{n}}=\mathbf{n}-\mathbf{g} \hat{n}_{1}
$$

is the combined noise under $\mathcal{H}_{1}$ and has the conditional distribution $C N\left(\mathbf{g} m_{1}, \mathbf{R}_{\tilde{\mathbf{n}} \tilde{\mathbf{n}}}=\sigma_{n}^{2} \mathbf{g g}{ }^{H}+\sigma_{n}^{2} \mathbf{I}\right)$ given $\hat{m}_{1}$. The argument $\omega$ has been dropped to simplify the notation. Here, $\mathbf{z} \sim C N(\boldsymbol{\mu}, \boldsymbol{\Sigma})$ means that the complex vector $\mathbf{z}$ is normal with mean $\boldsymbol{\mu}$ and covariance matrix $\boldsymbol{\Sigma}$.

\section{RECEIVER DESIGN}

Our aim in this section is to derive an optimal linear detector for the binary hypothesis test in (7). Linear detectors result in discriminant functionals which are linear in the data, and hence are easy to compute. The output of the discriminant functional is called a test statistic. Unlike Bayesian discriminant functionals which require knowledge of the probability distributions of the data, linear discriminant functionals require only knowledge of the first- and second-order statistics of the data.

The test statistic $\lambda$, obtained by using a linear discriminant functional of the form $D(\cdot)=\langle\cdot, \mathbf{w}\rangle$ for the binary hypothesis test in $(7)$, is

$$
\lambda:=D(\mathbf{m})=\langle\mathbf{m}, \mathbf{w}\rangle .
$$

Here $\mathbf{w}$ is a vector of $n-1$ receiver functions (template functions) that is designed to optimize a measure of detectability, and $\langle\cdot, \cdot\rangle$ is the inner product between two $(n-1)$-dimensional vectors, whose elements are in $L^{2}$.

A common measure of detectability is the SNR of the test statistic, which is defined as ${ }^{12}$

$$
\mathrm{SNR}_{\lambda}=\frac{\mathrm{E}\left[\lambda \mid \mathcal{H}_{1}, \hat{m}_{1}\right]-\mathrm{E}\left[\lambda \mid \mathcal{H}_{0}\right]}{\sqrt{0.5 \operatorname{Var}\left[\lambda \mid \mathcal{H}_{1}, \hat{m}_{1}\right]+0.5 \operatorname{Var}\left[\lambda \mid \mathcal{H}_{0}\right]}}
$$

The template vector or receiver vector $\mathbf{w}$ that maximizes $S N R_{\lambda}$ is known to be ${ }^{12}$

$$
\mathbf{w}=\mathbf{R}^{-1} \mathbf{g} \hat{m}_{1} .
$$

The performance of the detector is usually evaluated by plotting the receiver operating characteristics (ROC), which is a plot of probability of detection $P_{\mathrm{d}}=\mathcal{P}\left(\lambda>\gamma \mid \mathcal{H}_{1}, \hat{m}_{1}\right)$ versus probability of false alarm $P_{\mathrm{fa}}=\mathcal{P}(\lambda>$ $\gamma \mid \mathcal{H}_{0}$ ), obtained by varying the decision threshold $\gamma$. The area under the ROC curve (AUC) is a measure of the degree of overlap between the probability density functions of the test statistics under the two hypothesis (target and no target). 
If we use a pair of receivers, say receivers $i$ and 1, for detection then the binary hypothesis test in (7) reduces to

$$
\begin{aligned}
& \mathcal{H}_{1}: \quad \hat{m}_{i}=\frac{\hat{g}\left(\mathbf{x}_{i}, \mathbf{y}_{o}, \omega\right)}{\hat{g}\left(\mathbf{x}_{1}, \mathbf{y}_{o}, \omega\right)} \hat{m}_{1}+\hat{\tilde{n}} \\
& \mathcal{H}_{0}: \quad \hat{m}_{i}=\hat{n}_{i} \text {, }
\end{aligned}
$$

and the optimal linear receiver in (12) becomes

$$
w_{i}=\left(\frac{1}{\sigma_{n}^{2}\left|\frac{\hat{g}\left(\mathbf{x}_{i}, \mathbf{y}_{o}, \omega\right)}{\hat{g}\left(\mathbf{x}_{1}, \mathbf{y}_{o}, \omega\right)}\right|^{2}+\sigma_{n}^{2}}\right) \frac{\hat{g}\left(\mathbf{x}_{i}, \mathbf{y}_{o}, \omega\right)}{\hat{g}\left(\mathbf{x}_{1}, \mathbf{y}_{o}, \omega\right)} \hat{m}_{1} .
$$

If the propagation in the scattering environment is dominated by the direct path, the scattering environment may be approximated by the so called free-space model. In such a case the Green's function $\hat{g}\left(\mathbf{x}_{i}, \mathbf{y}_{o}, \omega\right)$ is given by

$$
\hat{g}\left(\mathbf{x}_{i}, \mathbf{y}_{o}, \omega\right)=\frac{1}{4 \pi\left|\mathbf{x}_{i}-\mathbf{y}_{o}\right|} e^{-j \frac{\omega}{c}\left|\mathbf{x}_{i}-\mathbf{y}_{o}\right|} .
$$

In this case, (14) is further simplified and the optimal receiver filter is a complex multiple of $m_{1}$. This complex factor, $\alpha$, depends on the noise variance and the difference in travel time from the target to receivers $i$ and 1 . Thus, the receiver is a delay-correlator; a filter that correlates the radar measurement $m_{i}(t)$ with an appropriately delayed version of $m_{1}(t)$. The receiver output $z\left(\tau_{i}\right)$ is

$$
z\left(\tau_{i}\right)=\alpha\left\langle m_{i}(t), T_{\tau_{i}} m_{1}(t)\right\rangle=\alpha \int m_{i}(t) \overline{m_{1}\left(t-\tau_{i}\right)} d t,
$$

where is the delay, $\tau_{i}=\frac{\omega}{c}\left(\left|\mathbf{x}_{i}-\mathbf{y}_{o}\right|-\left|\mathbf{x}_{1}-\mathbf{y}_{o}\right|\right), T_{\tau_{i}}$ is the delay operator

$$
T_{\tau_{i}} x(t)=x\left(t-\tau_{i}\right)
$$

and $\alpha=\left|\mathbf{x}_{1}-\mathbf{y}_{o}\right| /\left|\mathbf{x}_{i}-\mathbf{y}_{o}\right|$. Since, the incident field, $\hat{E}^{\text {in }}$, is the same in each measurements taken at each receiver, the delay-correlator is able to suppress the multipath that originates from the path from the transmitter to the target.

Value of $\tau_{i}$ for which (16) is maximum, does not uniquely identify the target location $\mathbf{y}_{o}$; rather it identifies a hyperbola on which the target lies. This hyperbola is the set of all points for which the difference in signal travel time to receivers $i$ and 1 is the same. The delay-correlator receiver for different pairs of receive antennas result in different hyperbola. The target location may be determined by intersecting the hyperbolas corresponding to different antenna pairs. One way to find the intersection is to form a geometric (or arithmetic) average of the images obtained from different delay-correlator receivers. Alternatively, a narrow window may be applied to the measurement $m_{i}(t)$ and $m_{1}(t)$ before calculating (16). The window is centered around the travel time from the target location $y_{o}$. This windowing approach is used in Sections 4.2 and 5.2.

\section{EXPERIMENT WITH REAL DATA}

\subsection{Experimental Setup}

A rough sketch of the scene setup is shown in Fig. 1. Three targets (different consumer vehicles) were present in the scene, approximately 50ft apart from each other, travelling along the road shown in Fig. 1. The middle vehicle's location was tracked with GPS measurements taken every $0.2 \mathrm{sec}$. The timestamps on the GPS measurements were used to synchronize the readings with the radar measurements.

Two transmit antennas and two receiver arrays, each consisting of three receive antennas, were used in the data collection. The transmitter/receiver geometry is shown in Fig. 2. Each transmitter had a 60 degrees beamwidth and was pointed towards the road. A series of five continuous wave $(\mathrm{CW})$ pulses, each of duration 20ns, were transmitted every 10sec (frame), with a $10 \mu \mathrm{sec}$ interval between each pulse transmission (see Fig. 3). 


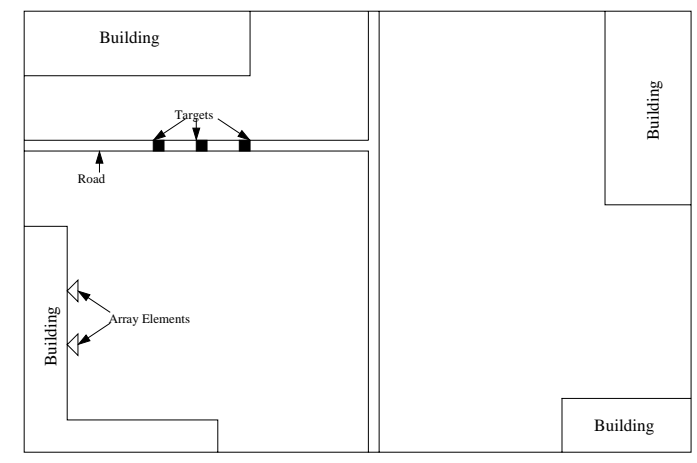

Figure 1. Sketch of the scene layout used for the experiment.

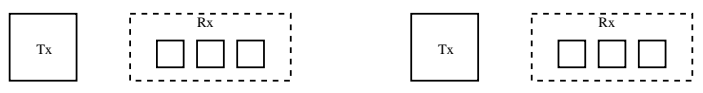

Figure 2. Transmitter/receiver geometry. Each array element in Fig. 1 corresponds to a Tx/Rx pair shown.

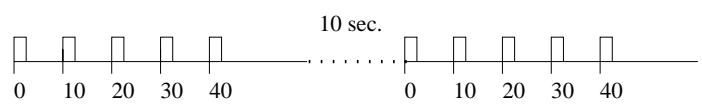

Figure 3. A series of five 20ns CW pulses are transmitted at $10 \mu$ s interval in each 10 s frame. The scheduling is illustrated in the above figure.

\subsection{Experimental Results}

Figures 4(a) and 4(b) show a sample matched filter image and image formed by the delay-correlator, respectively, along with a graph of the intensity profile taken along the road. Four consecutive 10-second frames, in which the targets were in view on the road approaching the intersection, were used for analysis. For each location in the matched filter image we determined the travel time to each antenna, and summed the corresponding values of the matched filter output. The delay-correlator image was formed by following the model defined by (16) and summing the results for each correlation. Each pixel in the images is considered as the value of the test statistic at the corresponding spatial location. The location of the target is indicated by the "O" on the images and the graphs in Fig. 4. The black line indicates the location of the road and the white lines are approximate locations of some of the building in the scene.

For the assessment of detection performance, the middle vehicle was used as the sole target. This was because the GPS readings allowed for accurate calculation of the target location. The images were compared using SNR and AUC estimates. The SNR of the test statistic was calculated using the formula given in (11) which is equivalent to the detectability index. Under Gaussian noise assumptions, the AUC can be related to the SNR in the following way. ${ }^{13}$

$$
\mathrm{AUC}=1 / 2\left(1+\operatorname{erf}\left(\mathrm{SNR}_{\lambda} / 2\right)\right)
$$

The first- and second-order statistics for the background and targets were calculated by taking a small rectangular subimage around the target and at a fixed offset from the target. These subimages are indicated in Fig. 4 by white rectangles. The values for the SNR estimations are given in Table 1 along with the estimated AUC values and SNR improvement of proposed processing over the baseline in $\mathrm{dB}$ scale. The table shows that there is a noticeable improvement in SNR for detection using the proposed method. This indicates that template used for matching in the baseline receiver may not have been an accurate representation of the actual illumination signal. 

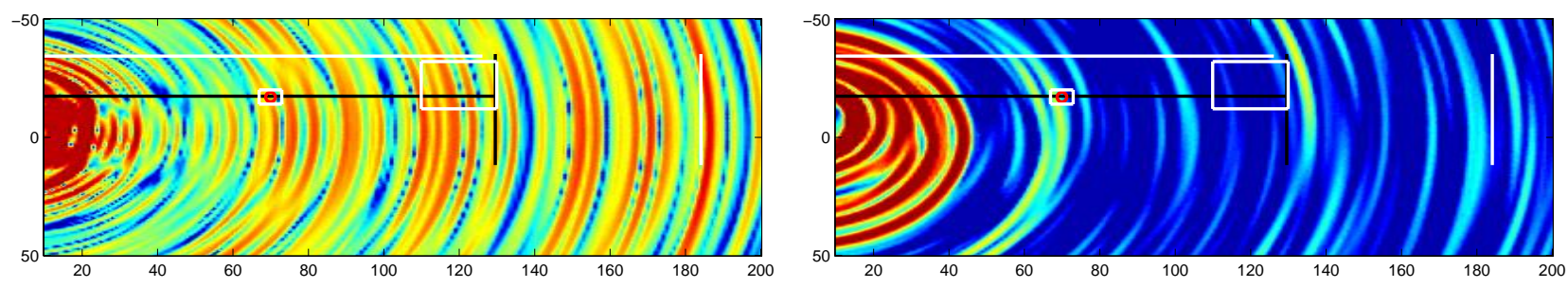

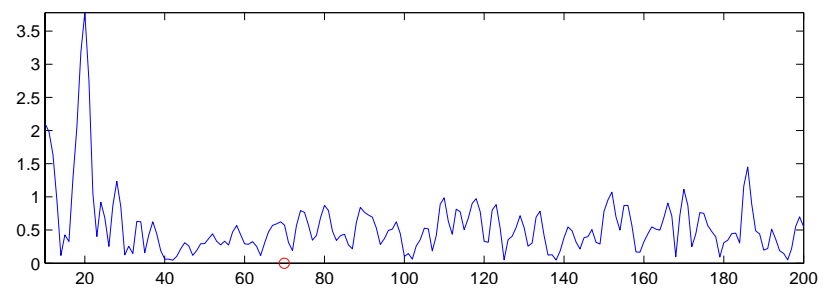

(a) The matched filter image

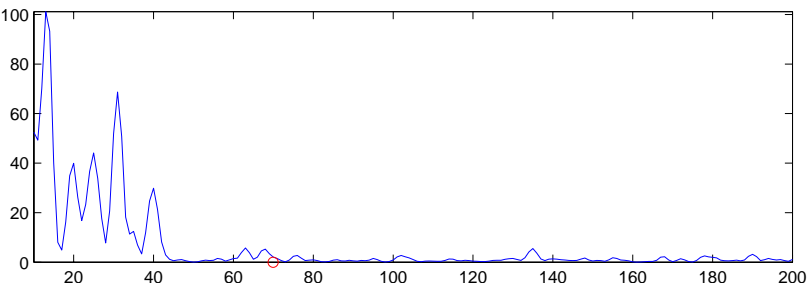

(b) The delay-correlator image

Figure 4. The two images compare the a) baseline matched filtering method and b) proposed delay-correlator method. Profiles of the $\lambda$ values taken along the road is also shown for each case.

\begin{tabular}{|c|l|c|c|c|}
\hline Frame No. & Method & SNR & AUC & Improv. (dB) \\
\hline \multirow{2}{*}{1} & Matched Filter & 1.0178 & 0.7641 & \multirow{2}{*}{7.9823} \\
& Delay-correlator & 2.5513 & 0.9644 & \\
\hline \multirow{2}{*}{2} & Matched Filter & 0.5277 & 0.6455 & \multirow{2}{*}{14.6222} \\
& Delay-correlator & 2.8413 & 0.9777 & \\
\hline \multirow{2}{*}{3} & Matched Filter & 0.3518 & 0.5982 & \multirow{2}{*}{6.4861} \\
& Correlation & 0.7424 & 0.7002 & \\
\hline \multirow{2}{*}{4} & Baseline & 0.2283 & 0.5641 & \multirow{2}{*}{16.6364} \\
& Delay-correlator & 1.5498 & 0.8634 & \\
\hline
\end{tabular}

Table 1. The SNR and AUC estimates are tabulated for four different time frames along with improvement over baseline in $\mathrm{dB}$.

\section{SIMULATION}

\subsection{Simulation Setup}

The simulated scene consisted of two parallel vertical plane walls and a ground, with a single transmitter and three receivers. No clutter was considered in this simulation. The data was generated by placing virtual sources wherever necessary via the method of images. The two walls were approximated as a rough surface by randomly displacing the placement of the walls repeatedly, forming a "cloud" of virtual sources as a result.

For the simulation, we considered a case where a simple CW waveform was transmitted. The multipaths from the ground and the walls however, distort portions of the returned signal from a true CW wave. Gaussian noise was added with enough power to overpower the signal as is typical in radar applications. The returned signal with and without noise is shown in figures $5(\mathrm{a})$ and $5(\mathrm{~b})$.

\subsection{Simulation Results}

The simulated data was processed in a similar manner to that described above for the real data using again, the matched filter and the delay-correlator (16). A sample of the simulation result for the matched filter and the delay-correlator are shown in figures $6(\mathrm{a})$ and $6(\mathrm{~b})$. The black "O" indicates the target location. The two walls are located at the top and bottom edges of the image plane.

The two receivers were again compared using the test statistics SNR (see (11)). However, using simulated data allows us to utilize multiple realization of the noise for the same scenario to arrive at a more reliable analysis. 


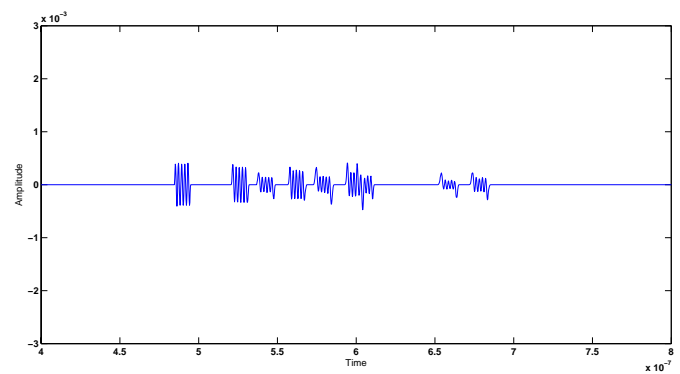

(a) The returned signal

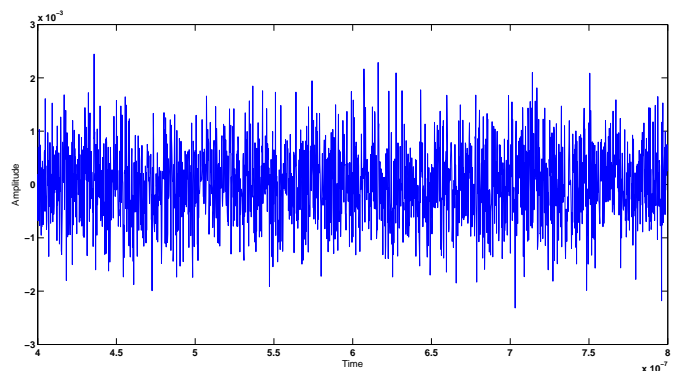

(b) The returned signal with noise

Figure 5. The presence of multipaths are clearly illustrated in a). The returned signal plus noise in b) shows that the received signal is noise dominated.

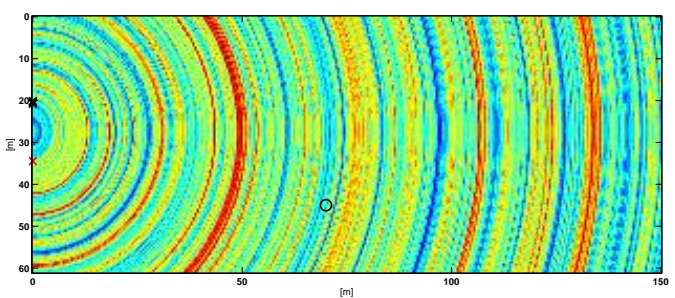

(a) Matched Filter

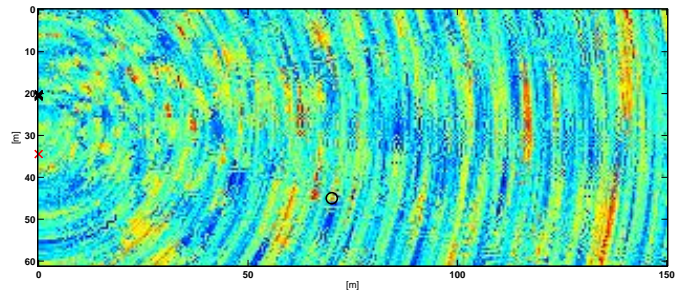

(b) Delay-correlator

Figure 6. The figure shows a sample of the baseline image formed using a) matched filtering and b) delay-correlator on the simulated data.

In all, 20 realizations of the data were used. The method of analysis was as follows. First a series of images were generated using the matched filter and delay-correlator methods. Two sets of images were generated for each receiver method, one with the target present and one without. These two sets of images were used to estimate the test statistics SNR with the images sets containing no target as the null hypothesis. The results are shown in Table 2.

\begin{tabular}{|l|c|c|c|}
\hline Method & SNR & AUC & Improv. (in dB) \\
\hline Matched Filter & 1.2983 & 0.8207 & 2.9852 \\
Delay-correlator & 1.8308 & 0.9023 & \\
\hline
\end{tabular}

Table 2. The SNR and AUC estimates are tabulated for the simulation results along with improvement over baseline in dB.

\section{CONCLUSION}

A new receiver design for target detection in an urban environment was presented. The receiver was chosen as the optimal linear filter corresponding to a binary hypothesis test involving the radar measurements. Unlike the conventional matched filtering, in the proposed receiver design the radar measurements are not matched to the illumination template; rather they are matched to a transformed version of the measurement at one of the receive 
antennas. Preliminary experimental results obtained using a real data set suggest that the proposed receiver design can offer significant improvement in detection performance.

For the sake of simplicity in presenting the main ideas we used the distorted-wave Born approximation and a point scatterer model. Future work will investigate more general scattering and target models, as well as the advantages of sparse aperture configurations.

\section{ACKNOWLEDGEMENT}

This work would not have been possible without the generous support from Robert J. Bonneau who provided the data used in this paper and initial insight into the problem.

We are grateful to Air Force Office of Scientific Research* (AFOSR) and the Defense Advanced Research Projects Agency (DARPA) for supporting this work under the agreements FA9550-04-1-0223, FA9550-06-1-0017, and FA8750-05-2-0285.

\section{REFERENCES}

1. E. H. Attia and B. D. Steinberg, "Self-cohering large antenna arrays using the spatial correlation properties of radar clutter," IEEE Transactions on Antennas and Propagation 37, pp. 30-38, January 1989.

2. H. D. Griffiths and C. J. Baker, "Passive coherent location radar systems. part 1: Performance prediction," IEE Proceedings of Radar, Sonar and Navigation 152, pp. 153-159, June 2005.

3. C. J. Baker, H. D. Griffiths, and I. Papoutsis, "Passive coherent location radar systems. part 2: Waveform properties," IEE Proceedings of Radar, Sonar, and Navigation 152, pp. 160-168, June 2005.

4. K. S. Kulpa, "Multi-static entirely passive detection of moving targets and its limitations," IEE Proceedings of Radar, Sonar, and Navigation 152, pp. 169-173, June 2005.

5. S. J. Norton, B. J. Carr, and A. J. Witten, "Passive imaging of underground acoustic sources," The Journal of the Acoustical Society of America 119, pp. 2840-2847, May 2006.

6. E. Larose, C. Montaldo, A. Derode, and M. Campillo, "Passive imaging of localized reflectors and interfaces in open media," Applied Physics Letters 88, pp. 104103-1-10413-3, 2006.

7. R. Snieder, "Extracting the green's function from the correlation of coda waves: A derivation based on stationary phase," Physical Review E 69, pp. 046610-1-046610-8, 2004.

8. A. Tolstoy, "Sensitivity of matched field processing to sound-speed profile mismatch for vertical arrays in a deep water pacific environment," Journal of Acoustic Society of America 85, pp. 2394-2404, June 1989.

9. J. L. Krolik, "The performance of matched-field beamformers with mediterranean vertical array data," IEEE Transactions on Signal Processing 44, pp. 2605-2611, October 1996.

10. R. J. Bonneau, "A rate distortion method for waveform design in rf target detection," in Aerospace Conference, 2006 IEEE, pp. 1-12, IEEE, 2006.

11. J. H. Taylor, Scattering Theory, Wiley, New York, NY, 1972.

12. R. O. Duda, P. E. Hart, and D. G. Stork, Pattern Classification, Wiley-Interscience, 2001.

13. H. L. Van Trees, Detection, Estimation, and Modulation Theory I: Detection, Estimation, and Linear Modulation Theory, vol. 1, Wiley-Interscience, 2001.

\footnotetext{
${ }^{*}$ Consequently the US Government is authorized to reproduce and distribute reprints for governmental purposes notwithstanding any copyright notation thereon. The views and conclusions contained herein are those of the authors and should not be interpreted as necessarily representing the official policies or endorsements, either expressed or implied, of the Air Force Research Laboratory or the US Government.
} 